\title{
DEPOLARIZATION STUDIES OF RADIO SOURCES AND THE UNIFIED SCHEME
}

\author{
S.T. GARRINGTON AND G.F. HOLMES \\ The University of Manchester, NRAL Jodrell Bank, UK \\ AND \\ D.J. SAIKIA \\ NCRA, TIFR, Pune, India
}

\section{Introduction}

In powerful FRII radio galaxies and quasars the polarization of the two radio lobes is often very asymmetric and strongly correlated with jet sidedness. These sources usually have one-sided jets and the lobe on the jet side depolarizes more slowly than the one on the counter-jet side (Laing 1988; Garrington et al. 1988). This correlation can be interpreted in terms of depolarization of the receding lobe by a halo of hot gas surrounding the radio source and is in the expected sense if the jet asymmetry is due to Doppler boosting.

\section{The FRI sources}

Low luminosity FRI sources have diffuse outer radio lobes or plumes. Their jets are reasonably symmetric on larger scales but often asymmetric closer to the nucleus. While the outer plumes may be transonic (Bicknell et al. 1990), several of the VLBI scale jets have moderately relativistic velocities (Giovannini, these proceedings). We have observed a sample of low- and intermediate-luminosity sources with the VLA at $\lambda 6$ and $18 / 20 \mathrm{~cm}$ at matched resolutions of 5 or 15 arcsec. Larger sources were also observed with the WSRT at $\lambda 49 \mathrm{~cm}$.

Many sources (large and small) show little depolarization between $\lambda 6$ and $18 / 20 \mathrm{~cm}$. Several sources show significant, symmetric depolarization: these sources are predominantly 'fat' or twisted FRI sources. Several sources show strong asymmetric depolarization, with less depolarization on the side 

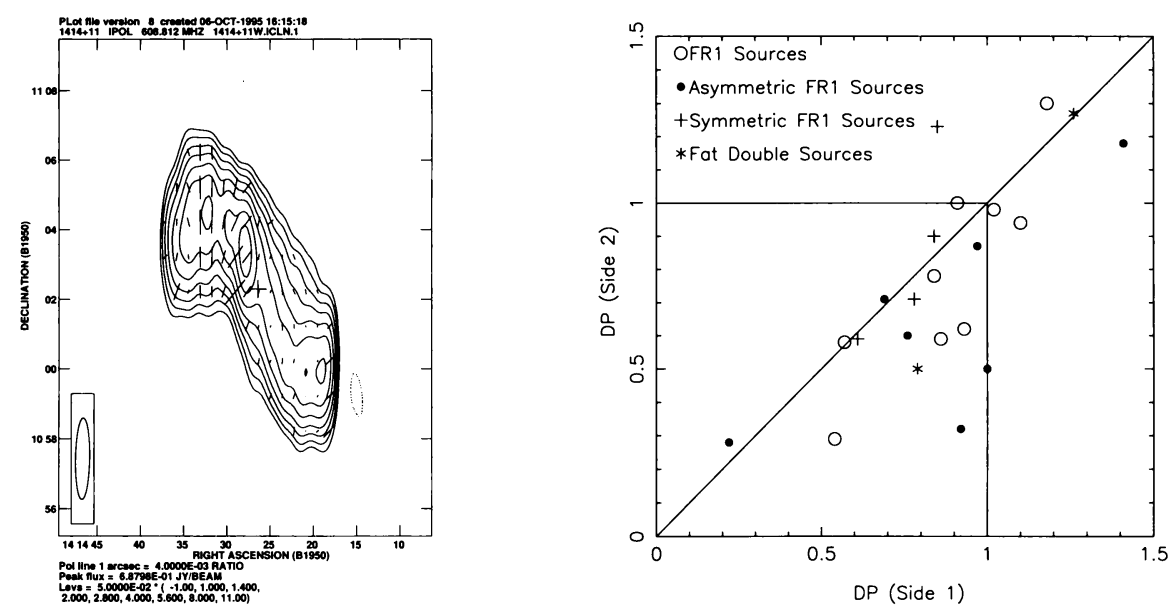

Figure 1. Left: the radio galaxy $3 \mathrm{C} 296$ observed with WSRT at $\lambda 49 \mathrm{~cm}$. The southern jet, which is much fainter in higher resolution maps, depolarizes between 18 and $49 \mathrm{~cm}$.

Figure 2. Right: plot of DP $\left(=m_{18} / m_{6}\right.$ or $\left.=m_{49} / m_{18}\right)$ for the two jets or lobes. Side 1 has the brighter jet at high resolution.

of the source with the brighter jet. These sources include the fat doubles and FRI sources with the more asymmetric jets. In 3C296 (Figure 1), the depolarization asymmetry becomes very pronounced in the WSRT $49 \mathrm{~cm}$ map. Figure 2 compares the measured depolarization ratio (low values of DP correspond to stronger depolarization) for the two sides of each source: The correlation between jet sidedness and depolarization asymmetry is seen in the cluster of sources below the diagonal line and is in the same sense as the more powerful sources.

Our provisional results suggest that FRI sources have low Faraday dispersion, and are generally more symmetric in depolarization than FRII sources. The depolarization asymmetry seen in the sources with the most asymmetric jets is consistent with the view that these jets may be initially relativistic.

\section{The FRII radio galaxies and quasars}

In the unified model for FRII radio galaxies and quasars (eg Barthel 1989), we would expect the radio galaxies to show the same correlation of depolarization with jet-sidedness, but perhaps at a lower level. We observed a sample of radio galaxies, selected from the $3 \mathrm{CR}$ complete sample, with the WSRT at $\lambda 49$ and $21 \mathrm{~cm}$ and with the VLA at $\lambda 20,18$ and $6 \mathrm{~cm}$. Combining these with data from the literature, we find that 19 out of 27 radio galaxies with jets show stronger depolarization on the counter-jet side. The weaker trend seen for radio galaxies appears consistent with the unified scheme. 
Since the degree of depolarization asymmetry is stronger at higher redshift and jets are rare in distant radio galaxies, we also constructed a smaller matched sample of 9 radio galaxies and 9 quasars in the redshift range of about 0.3 to 1 . The numbers of sources with stronger depolarization on the counter-jet side is $8 / 9$ for the quasars and $5 / 9$ for the radio galaxies (although the most asymmetric cases are in the expected sense). It appears that in radio galaxies the correlation of depolarization with lobe separation is stronger than the relation with jet sidedness, while the reverse is true for quasars (c.f. Laing 1993). While the correlation of depolarization with armlength may be related to an intrinsic asymmetry in the distribution of gas surrounding the source (McCarthy et al 1991), it would also be a natural consequence of a symmetric, centrally peaked distribution of depolarizing gas.

\section{Other sources}

A few per cent of radio-loud quasars are highly asymmetric with the brightness ratio of the lobes exceeding a few 100:1. This may be due to Doppler boosting (Saikia et al. 1990). We have observed 13 of these asymmetric quasars with the VLA at $\lambda 20$ and $6 \mathrm{~cm}$. The new maps reveal weak counterlobes in some cases, and show that the 'core' is an unrelated source in others. The brighter lobes show little depolarization between $\lambda 20$ and $6 \mathrm{~cm}$, consistent with them being the approaching components of double-lobed sources seen through little of the depolarizing medium of the host galaxy or cluster. Some of the smaller sources, however, have low values of polarization at both $\lambda 20$ and $6 \mathrm{~cm}$.

From multifrequency studies of the cores of core-dominated quasars, we find that the rotation measures (RM) are generally less than about 30 $\mathrm{rad} \mathrm{m}^{-2}$ at $\lambda>6 \mathrm{~cm}$, but extend up to a few hundred $\mathrm{rad} \mathrm{m}^{-2}$ at shorter wavelengths, perhaps due to contributions from components buried deeper in the core. There is some evidence that the cores of galaxies are much more weakly polarized than quasars (Saikia, Singal \& Cornwell 1987), perhaps due to depolarization by the obscuring torus required by unified models.

\section{References}

Barthel P.D. 1989, $A p J$, 336, 606.

Bicknell G.V. et al., 1990, $A p J, 354,98$.

Garrington S.T, et al., 1988, Nature, 331, 147.

Laing R.A., 1988, Nature, 331, 149.

Laing R.A., 1993, in Astrophysical Jets, eds Burgarella D., et al., CUP, p. 95

McCarthy, P.J., van Breugel, W. and Kapahi,V.K, 1991, ApJ, 371,478.

Saikia D.J., Junor W., Cornwell T.J., Muxlow T.W.B., Shastri P., 1990, MNRAS, 245, 408.

Saikia D.J., Singal A.K., Cornwell T.J., 1987, MNRAS, 224, 379. 